\title{
Quantitative and Pattern Recognition Analyses for the Quality Evaluation of Cimicifugae Rhizoma by HPLC
}

\author{
Zhe Fang, Dong Cheul Moon, ${ }^{\dagger}$ Kun Ho Son, ${ }^{\ddagger}$ Jong Keun Son, ${ }^{\S}$ Byung Sun Min, and Mi Hee Woo* \\ College of Pharmacy, Catholic University of Daegu, Gyeongsan 712-702, Korea. E-mail: woomh@cu.ac.kr \\ ${ }^{\dagger}$ College of Pharmacy, Chungbuk National University, Cheongju 361-763, Korea \\ ${ }^{\star}$ College of Life Science, Andong National University, Andong 760-740, Korea \\ ${ }^{\S}$ College of Pharmacy, Yeungnam University, Gyeongsan 712-749, Korea \\ Received July 6, 2010, Accepted November 15, 2010
}

\begin{abstract}
In this study, quantitative and pattern recognition analysis for the quality evaluation of Cimicifugae Rhizoma using $\mathrm{HPLC} / \mathrm{UV}$ was developed. For quantitative analysis, three major bioactive phenolic compounds were determined. The separation conditions employed for HPLC/UV were optimized using ODS $\mathrm{C}_{18}$ column $(250 \times 4.6 \mathrm{~mm}, 5 \mu \mathrm{M})$ with isocratic elution of acetonitrile and water with $0.1 \%$ phosphoric acid as the mobile phase at a flow rate of $1.0 \mathrm{~mL} / \mathrm{min}$ and a detection wavelength of $323 \mathrm{~nm}$. These methods were fully validated with respect to the linearity, accuracy, precision, recovery, and robustness. The HPLC/UV method was applied successfully to the quantification of three major compounds in the extract of Cimicifugae Rhizoma. The HPLC analytical method for pattern recognition analysis was validated by repeated analysis of twelve reference samples corresponding to five different species of Cimicifugae Rhizoma and seventeen samples purchased from markets. The results indicate that the established HPLC/UV method is suitable for the quantitative analysis and quality control of multi-components in Cimicifugae Rhizoma.
\end{abstract}

Key Words: Cimicifugae Rhizoma, HPLC, Pattern recognition, Quality control

\section{Introduction}

Herbal medicines have a long history in therapeutic field and they are attracting considerable attention because of low toxicity and excellent therapeutic benefit. Quality control for herbal drugs is difficult than for synthetic drugs because of the chemical complexity of the ingredients. In herbal drugs, no single active constituent is responsible for the overall pharmacological efficacy. For quality control of complex systems, the determination of only a few compounds cannot give a comprehensive and accurate assessment of all active compounds in herbal drugs. Pattern recognition analysis can provide the information of overall chemical composition of herbal medicines traditionally used for quality control. ${ }^{1}$

Cimicifugae Rhizoma is the root of Cimicifuga heracleifolia Komarov or the other species in the Korean Pharmacopeia (K.P.), of C. simplex Wormskjord, C. dahurica Maximmowicz, C. foetida Linne, or C. heracleifolia Komarov in the Japanese Pharmacopoeia (J.P.), and of C. hercleifolia Komarov, C. dahurica Maxim., or $C$. foetida L. in the Chinese Pharmacopoeia (C.P.). Cimicifugae Rhizoma is controlled to contain not less than $0.1 \%$ of isoferulic acid in C.P. There are ten different Cimicifuga species: C. heracleifolia, C. dahurica, C. foetida, C. simplex, C. japonica, C. acerina, C. biternat, C. racemosa, C. americana and $C$. rubifolia. We collected five different species of Cimicifugae Rhizoma for this study from Korea and China. Five different species included $C$. heracleifolia, C. dahurica, C. foetida and C. simplex, which have been used in K.P., J.P. and C.P., and C. japonica. Pharmacological studies have revealed in to have bone resorption inhibition, ${ }^{2}$ vasoactive effect, ${ }^{3}$ estrogenic activity, ${ }^{4}$ antiinflammatory, ${ }^{5}$ cytotoxicity, ${ }^{6}$ anti-microbial and cytokine modulation effect ${ }^{7}$ and anticomplement activity. $^{8}$
Some HPLC/UV analytical methods have been developed for the analysis of Cimicifugae Rhizoma and its related products. ${ }^{9-11}$ Kan He et al. reported the triterpene glycoside cimigenol 3-O-arabinoside, cimifugin and cimifugin-3-O-glucoside as specific markers for the distinction of $C$. racemosa from the other Cimicifuga species. ${ }^{11}$ Black cohosh, one of the most important herbal products in the US dietary supplements market, is manufactured from roots and rhizomes of C. racemosa. Cimicifugae Rhizoma samples used in this study didn't include $C$. racemosa which is native to the Eastern United States, growing as far south as Florida. Furthermore, these studies were focused only quantitative analysis of selected marker compounds which are not promising approaches for the quality control of finger printing analysis of herbal drugs. In the present study, a simple, sensitive and precise reverse-phase HPLC/UV method has been developed for the quantitative determination of three marker phenolic components, caffeic acid (1), ferulic acid (2) and isoferullic acid (3) along with pattern-recognition method for the quality control of Cimicifugae Rhizoma extract. The twelve Cimicifugae Rhizoma authentic samples collected from China and Korea and seventeen Cimicifugae Rhizoma samples purchased from the markets were analyzed by HPLC after extraction with 50\% ethanol. Cimicifugae Rhizoma samples exhibited very different triterpenoid chromatogram patterns in HPLC according to their different Cimicifuga species. Quantitative analysis of isoferulic acid single compound in C.P. would not be an adequate approach for quality control of Cimicifugae Rhizoma. Therefore in pattern recognition analysis we used three phenolic marker compounds as caffeic aicd (1), ferulic acid (2) and isoferulic acid (3) instead of triterpenoids. In pattern analysis with multivariate statistical analysis we used R-2.11.0 program (downloaded from web http://www.r-project.org) to analyze eleven authentic samples of Cimicifugae Rhizoma 
and seventeen commercial ones. Subsequent pattern analysis was applied to assess the comprehensive quality of Cimicifugae Rhizoma.

\section{Experimental Section}

Plant Material. Cimicifugae Rhizoma samples collected in 2006 for this study include the following accessions: a $C$. foetida (c8) collected from China, and four C. heracleifolia (c1 c4), two $C$. dahurica (c5 and c6), two $C$. foetida (c7 ana c9), two $C$. simplex (c10 and $\mathrm{c} 11)$ and a $C$. japonica $(\mathrm{c} 12)$ collected from Korea, and seventeen commercial Cimicifugae Rhizoma samples (c13 and c14 from Yangyeongsi, Daegu, Korea; c15 c19 from Gyongdong market, Korea; c20 c24, c26 and c27 from Yeongcheon market, Korea; c25 from Xining, China; c28, c29 from Seoul market, Korea) purchased from markets of Korea and China.

Reagents. All of the standard compounds were provided by Prof. Kun Ho Son, Andong National University, Andong, Korea. Their structures were unambiguously identified by NMR and MS data, with the published data, such as caffeic acid, ${ }^{12}$ ferulic acid $^{13}$ and isoferulic acid. ${ }^{14}$ The standard compound structures were shown in Fig. 1. Purity of standard compounds was estimated to be higher than $95 \%$ based on HPLC and LC-MS/MS analysis. Internal standard, 4-hydroxycinnamic acid (4), was purchased from Sigma Chemicals (St. Louis, MO, USA). Methanol and acetonitrile of HPLC grade were purchased from Merck K GaA (Darmstadt, Germany). All other chemicals used were of analytical grade unless otherwise noted. Distilled water was prepared using Milli-Q purification system (Millipore, Bedford, MA, USA).

Sample Preparation. To determine the content of three marker compounds and pattern recognition analysis of Cimicifugae Rhizoma samples, the dried rhizome powder were used for each extraction. Cimicifugae Rhizoma samples were powdered and sieved through 50 mesh, and about $0.3 \mathrm{~g}$ of the powder were accurately weighed and added $50 \mathrm{~mL}$ of $50 \%$ ethanol, accurately measured weight and refluxed for 4 hours at $80{ }^{\circ} \mathrm{C}$. The solution was cooled, weighed again, and made up the loss in weight with $50 \%$ ethanol. The solution was filtered through a $0.45 \mu \mathrm{M} \mathrm{mem-}$ brane filter and the filtrate was used as the test solution. Sample solution of $10 \mu \mathrm{L}$ was subjected to injection into the HPLC system.

HPLC/UV Condition. The HPLC equipment was a Waters HPLC system (Waters, Milford, MA, USA) with Waters 600 pumps, a Waters 486 UV detector and a Waters 717 autosampler. YMC ODS-H80 $(250 \times 4.6 \mathrm{~mm}, 4 \mu \mathrm{m})$, Shiseido capcell pak $(250 \times 4.6 \mathrm{~mm}, 5 \mu \mathrm{M})$ and Shodex ODS pak $(250 \times 4.6 \mathrm{~mm}$, $5 \mu \mathrm{M})$ columns were tested with the guard columns filled with the same stationary phase. A ( $100 \%$ acetonitrile $)$ and B $(0.1 \%$ phosphoric acid in water) were used as the mobile phase using a isocratic condition $(\mathrm{A}: \mathrm{B}=15: 85, \mathrm{v} / \mathrm{v} \%)$ to analyze samples. The mobile phase was filtered under vacuum through a $0.45 \mu \mathrm{M}$ membrane filter and degassed prior to use. The analysis was carried out at a flow rate of $1.0 \mathrm{~mL} / \mathrm{min}$ with the detection wavelength set to $323 \mathrm{~nm}$, and the total run time was $40 \mathrm{~min}$. All compounds could be resolved with baseline separation at $323 \mathrm{~nm}$ with the maximum absorption. Hence, characteristic chromato-

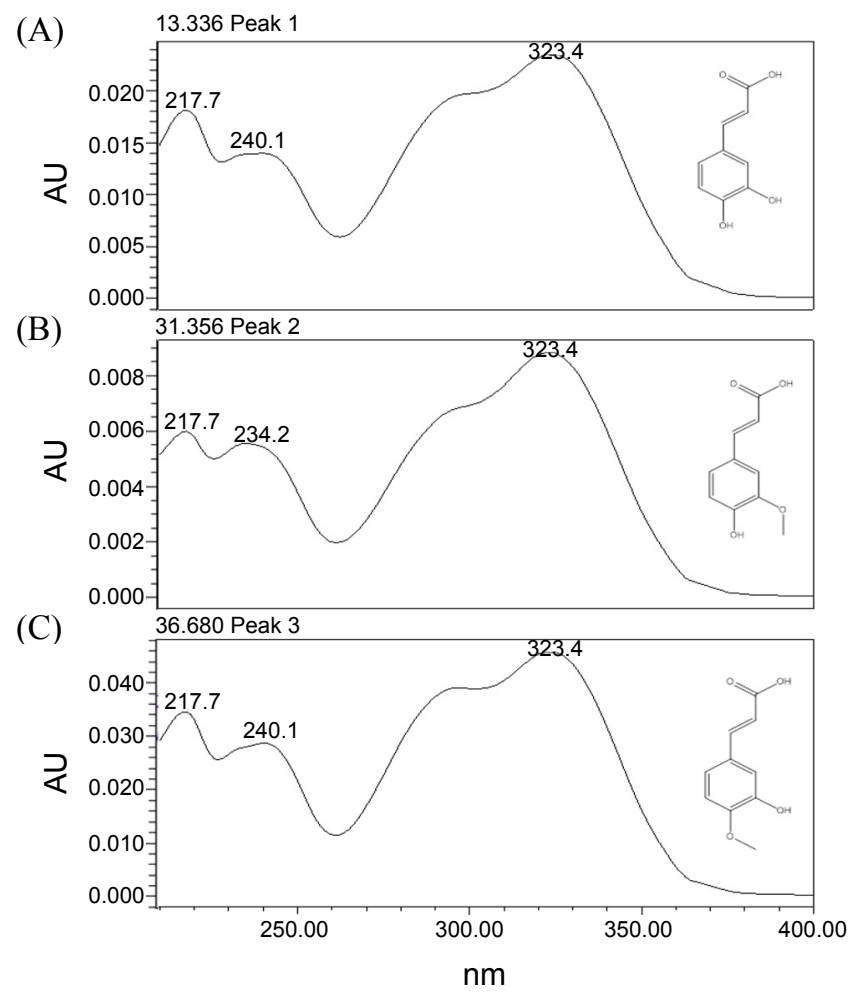

Figure 1. Chemical structure of marker compounds caffeic acid (A), ferulic acid (B) and isoferulic acid (C) along with UV spectra.

graphic patterns were obtained at $323 \mathrm{~nm}$. The chromatograms were processed using software Empower pro software, build 1154 (Waters, Milford, MA).

Analytical Method Validation. The standards (4 mg) of caffeic acid, ferulic acid and isoferulic acid were each accurately weighed and then dissolved with $10 \mathrm{~mL}$ of $100 \%$ methanol to produce stock standard solutions of $400 \mathrm{ppm}$, respectively. The internal standard (4-hydroxycinnamic acid) of $500 \mathrm{mg}$ was accurately weighed and then dissolved with $100 \mathrm{~mL}$ of $100 \%$ methanol to produce stock solution of 5,000 ppm. The calibration curves were made by diluting the stock solutions with $100 \%$ methanol. The reference solution of the three phenolic compounds at concentrations of $0.2 \sim 40 \mu \mathrm{g} / \mathrm{mL}$ was analyzed by HPLC/UV. The regression equations were calculated in the form of $y=a x+b$, where $y$ and $x$ correspond to peak area ratio for internal standard and compound concentration, respectively.

The recovery tests were executed by mixing a powdered sample $(0.3 \mathrm{~g})$ with three control levels $(20 \%, 50 \%$, and $100 \%$ concentrations of the each compound contained in the samples) of the reference compounds. The mixture was then extracted by reflux with $50 \mathrm{~mL}$ of $50 \%$ ethanol at $80{ }^{\circ} \mathrm{C}$ for 4 hours. The extract solution was filtered through a $0.45 \mu \mathrm{M}$ membrane. The $\mathrm{HPLC} / \mathrm{UV}$ analysis experiments were performed in triplicate for each control level. The data was compared with those from the standard solution and extracted sample. Precision and accuracy were determined by multiple analysis $(n=5)$ of quality control samples prepared at lower, medium and higher concentrations spanning the calibration range $(0.2,20$, and $40 \mu \mathrm{g} / \mathrm{mL})$.

Pattem Recognition Analysis. The twelve authentic samples 
of Cimicifugae Rhizoma were chosen as references for the quality control of Cimicifugae Rhizoma. To evaluate the phytochemical equivalency among the twenty-nine samples corresponding to twelve authentic and seventeen commercial ones, pattern recognition analysis was conducted. In this study we used three marker compound peaks (caffeic acid (1), ferulic acid (2) and isoferulic acid (3)) for pattern recognition analysis. Pattern recognition analysis was conducted using software package R-2.11.0.

\section{Results and Discussion}

Optimization of Chromatographic Condition. The HPLC conditions were selected by the requirement for obtaining the chromatograms with a better resolution of the adjacent peaks within a short retention time. For the optimization of chromatographic condition, the effect of the composition of mobile phase on the separation was examined. Mobile phase of watermethanol did not result in the satisfactory separation of structurally similar compounds. Acetonitrile as an organic modifier demonstrated a significant improvement on separation. We had tested the addition of $0.1 \%, 1 \%$ and $10 \%$ acid (acetic acid, formic acid and phosphoric acid) to the mobile phase to do experiment. The addition of $0.1 \%$ phosphoric acid to the mobile phase to all of the compounds resulted in a good resolution, as well as satisfactory peak symmetry and shape. The typical chromatograms of samples and standard mixture are shown in Fig. 2, from which one can observe that all target compounds and an internal standard are completely separated within 40 minutes. 4-Hydroxycinnamic acid (4) was selected as an internal standard. The chromatographic peaks of the analytes in sample solution were identified by comparing their retention times with those of the reference standards and further confirmed by spiking samples with the reference compounds (Fig. 2). For the choice of detection wavelength, extract sample was scanned between 200 - $400 \mathrm{~nm}$ using PDA detector. All compounds

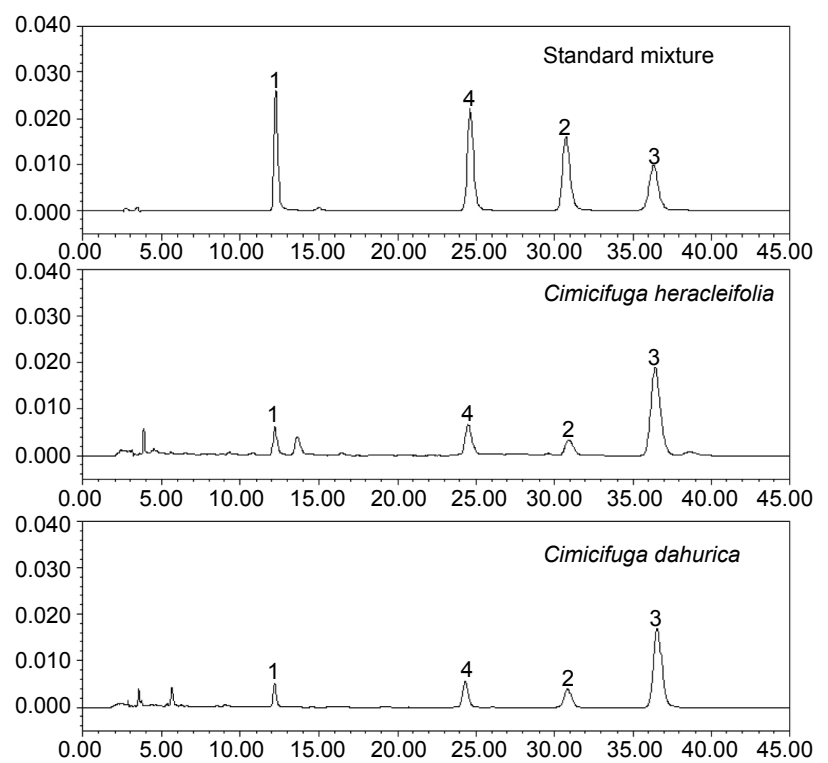

could be resolved with baseline separation at $323 \mathrm{~nm}$ with the maximum absorption shown for three major constituents (Fig. 1). Hence, characteristic chromatographic patterns were obtained at $323 \mathrm{~nm}$.

Optimization of Sample Preparation Condition. Nine extracting solvents, $100 \%$ ethanol, $75 \%$ ethanol, $50 \%$ ethanol, $25 \%$ ethanol, $100 \% \mathrm{H}_{2} \mathrm{O}, 100 \%$ methanol, $75 \%$ methanol, $50 \%$ methanol and $25 \%$ methanol were compared with regard to sample assays using reflux extraction for 4 hours at $80^{\circ} \mathrm{C}$. When sample was extracted with $50 \%$ ethanol, the sample assay was higher than the other solvent samples. Therefore, we employed 50\% ethanol as an extracting solvent throughout this work. Two extraction methods, ultra-sonication and reflux using 50\% ethanol extraction solvent, were compared with regard to sample assays. When used for reflux extraction method, the sample assay was higher than sonication one. To determine the time needed to obtain complete extractions, extractions of a sample were performed for six different lengths of time (1, 2, 3, 4, 5 and 6 hours). The rest of the variables employed were: $50 \%$ ethanol solvent and reflux extraction method at $80{ }^{\circ} \mathrm{C}$. When extraction time was 4 hours, the sample assay was same as 5 and 6 hours, and higher than 1, 2 and 3 hours. Therefore, when extraction time was 4 hours, all of the compounds were sufficiently extracted.

Validation. Each coefficient of correlation $\left(r^{2}\right)$ was $>0.999$, as determined by least square analysis, suggesting good linearity between the peak area ratio and the compound concentrations (Table 1). The limits of detection (LOD) and limits of quantitation (LOQ) were evaluated based on the lowest detectable peak in the chromatogram having a signal-to-noise $(\mathrm{S} / \mathrm{N})$ ratio of 3 and 10, respectively. Under our experimental conditions, we listed LOD and LOQ in Table 1. The obtained values for both LOD and LOQ for these three standards were shown to be low enough to detect traces of these compounds in either crude extract or its preparation.

The extraction recovery test was performed by extracting a

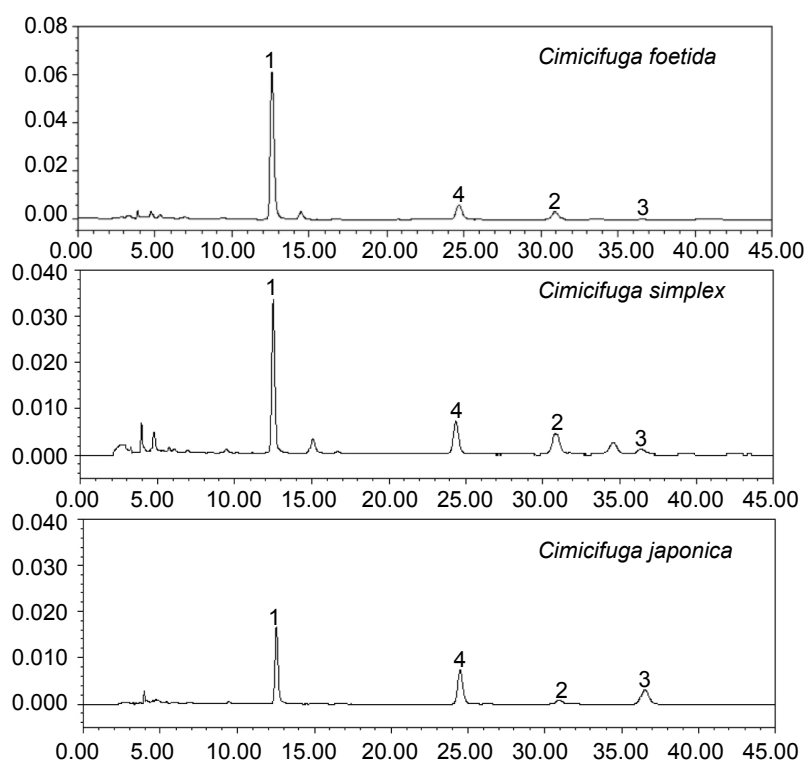

Figure 2. HPLC chromatograms of standard mixture and five different species of Cimicifugae Rhizoma. 1 caffeic acid, 2 ferulic acid, $\mathbf{3}$ isoferulic acid, 4 4-hydroxycinnamic acid (I.S.). 
Table 1. Calibration graphs, linear ranges, LOD and LOQ

\begin{tabular}{lcccccc}
\hline \multicolumn{1}{c}{ Analytes } & $\begin{array}{c}\text { linear range } \\
(\mu \mathrm{g} / \mathrm{mL})\end{array}$ & Slope (a) & Intercept (b) & $\begin{array}{c}\text { Correlation } \\
\text { coefficient (r) }\end{array}$ & LOD (ng/mL) & LOQ (ng/mL) \\
\hline Caffeic acid (1) & $0.2 \sim 40$ & 0.2710 & 0.0105 & 0.9999 & 12 & 36 \\
Ferulic acid (2) & $0.2 \sim 40$ & 0.2680 & 0.0190 & 0.9997 & 13 & 39 \\
Isoferulic acid (3) & $0.2 \sim 40$ & 0.2494 & 0.0277 & 0.9999 & 15 & 45 \\
\hline
\end{tabular}

Table 2. Recovery of marker compounds through standard addition $(\mathrm{n}=5)$

\begin{tabular}{|c|c|c|c|c|}
\hline Analyte & Fortified conc. $(\mu \mathrm{g} / \mathrm{mL})$ & Observed conc. $(\mu \mathrm{g} / \mathrm{mL})$ & Recovery mean (\%) & Recovery cv (\%) \\
\hline \multirow{4}{*}{ Caffeic acid (1) } & 0 & 3.03 & - & - \\
\hline & 1.5 & 4.57 & 102.88 & 0.17 \\
\hline & 3.0 & 5.98 & 98.39 & 0.11 \\
\hline & 4.5 & 7.30 & 94.97 & 0.07 \\
\hline \multirow{4}{*}{ Ferulic acid (2) } & 0 & 4.11 & - & - \\
\hline & 2.0 & 6.14 & 101.63 & 0.07 \\
\hline & 4.0 & 8.00 & 97.18 & 0.12 \\
\hline & 6.0 & 9.88 & 96.23 & 0.10 \\
\hline \multirow{4}{*}{ Isoferulic acid (3) } & 0 & 18.70 & - & - \\
\hline & 4.0 & 22.58 & 97.05 & 0.35 \\
\hline & 8.0 & 26.95 & 103.14 & 0.41 \\
\hline & 16.0 & 34.66 & 99.77 & 0.44 \\
\hline
\end{tabular}

Table 3. Precision and accuracy of analytical results

\begin{tabular}{|c|c|c|c|c|c|c|c|c|c|}
\hline \multirow[b]{2}{*}{ Analyte } & \multirow{2}{*}{$\begin{array}{l}\text { Nominal } \\
\text { conc. } \\
(\mu \mathrm{g} / \mathrm{mL})\end{array}$} & \multicolumn{4}{|c|}{ Intraday $(\mathrm{n}=5)$} & \multicolumn{4}{|c|}{ Inter-day $(\mathrm{n}=5)$} \\
\hline & & $\begin{array}{c}\text { Observed } \\
(\mu \mathrm{g} / \mathrm{mL})\end{array}$ & SD & $\begin{array}{c}\text { Accuracy } \\
(\%)\end{array}$ & $\begin{array}{c}\text { Precision } \\
(\%)\end{array}$ & $\begin{array}{c}\text { Observed } \\
(\mu \mathrm{g} / \mathrm{mL})\end{array}$ & SD & $\begin{array}{c}\text { Accuracy } \\
(\%)\end{array}$ & $\begin{array}{l}\text { Precision } \\
\quad(\%)\end{array}$ \\
\hline \multirow{3}{*}{ Caffeic acid (1) } & 0.20 & 0.19 & 1.78 & 96.29 & 1.85 & 0.19 & 0.89 & 96.04 & 0.93 \\
\hline & 20.00 & 20.69 & 0.97 & 103.43 & 4.34 & 20.92 & 2.00 & 104.60 & 2.09 \\
\hline & 40.00 & 41.07 & 2.48 & 102.68 & 2.45 & 40.69 & 1.53 & 101.72 & 1.51 \\
\hline \multirow{3}{*}{ Ferulic acid (2) } & 0.20 & 0.21 & 2.44 & 103.03 & 2.37 & 0.19 & 1.96 & 97.14 & 2.03 \\
\hline & 20.00 & 19.53 & 1.37 & 97.63 & 1.41 & 19.59 & 1.64 & 97.94 & 1.68 \\
\hline & 40.00 & 40.95 & 1.00 & 102.38 & 0.98 & 41.22 & 1.52 & 103.05 & 1.48 \\
\hline \multirow{3}{*}{ Isoferulic acid (3) } & 0.20 & 0.19 & 2.32 & 98.57 & 2.35 & 0.19 & 1.50 & 98.33 & 1.53 \\
\hline & 20.00 & 19.25 & 3.60 & 96.23 & 3.74 & 19.10 & 2.94 & 95.48 & 3.09 \\
\hline & 40.00 & 42.75 & 2.71 & 106.88 & 2.54 & 42.60 & 2.70 & 106.49 & 2.53 \\
\hline
\end{tabular}

known amount of the three compounds from the Cimicifugae Rhizoma powder samples. A known amount of each standard compound at three levels was mixed with the sample powder and extracted with $50 \%$ ethanol, as described in the experimental section. The $\%$ recovery of each standard ranged from 94.97 to $103.14 \%$, and the RSD was less than $0.44 \%$ (Table 2 ). The average recovery was represented by the formula: $\mathrm{R}(\%)=$ [(amount from the sample spiked standard-amount from the sample)/amount from the spiked standard] $\times 100$. Precision and accuracy were determined by multiple analysis $(n=5)$ of quality control samples prepared at lower, medium and higher concentrations spanning the calibration range. Intra-assay precision and accuracy were determined from the variability of multiple analyses $(n=5)$ of quality control samples analyzed within the same analytical run. The remaining quality control samples had the intra-assay precision below $4.34 \%$ and accuracy between $96.23 \%$ and $106.88 \%$. Inter-assay precision and accuracy were evaluated from the variability of multiple analyses $(n=5)$ of quality control samples analyzed on single analytical run and extended for consecutive five days. The remaining quality control samples had the inter-assay precision lower than 3.09\% and accuracy between $95.48 \%$ and $106.49 \%$. The above data reflects that the developed method is highly reproducible and precision and accuracy data are presented in Table 3.

The robustness was determined in order to evaluate the reliability of the established HPLC methods. All of the parameters 
Table 4. Robustness of marker compounds through standard addition

\begin{tabular}{|c|c|c|c|c|c|c|c|}
\hline & \multicolumn{7}{|c|}{ Analytes } \\
\hline & \multicolumn{3}{|c|}{ caffeic acid } & \multicolumn{2}{|c|}{ ferulic acid } & \multicolumn{2}{|c|}{ isoferulic acid } \\
\hline & & mean & SD & mean & SD & mean & SD \\
\hline \multirow[t]{16}{*}{ Column } & \multicolumn{7}{|c|}{ Theoretical plate (N) } \\
\hline & YMC & 1121 & 128 & 4146 & 143 & 7214 & 209 \\
\hline & Shisiedo & 1001 & 48 & 2927 & 16 & 2677 & 125 \\
\hline & Sodex & 543 & 27 & 1834 & 98 & 2243 & 62 \\
\hline & \multicolumn{7}{|c|}{ Capacity factor $\left(k^{\prime}\right)$} \\
\hline & YMC & 3.62 & 0.04 & 8.88 & 0.10 & 10.39 & 0.12 \\
\hline & Shiseido & 3.43 & 0.16 & 7.56 & 0.29 & 8.92 & 0.35 \\
\hline & Sodex & 4.39 & 0.02 & 10.17 & 0.03 & 11.84 & 0.03 \\
\hline & \multicolumn{7}{|c|}{ Separation factor $(\alpha)$} \\
\hline & YMC & 0.48 & 0.01 & 1.17 & 0.02 & 1.16 & 0.00 \\
\hline & Shiseido & 0.45 & 0.02 & 1.00 & 0.03 & 1.18 & 0.00 \\
\hline & Sodex & 0.58 & 0.00 & 1.34 & 0.01 & 1.16 & 0.00 \\
\hline & \multicolumn{7}{|c|}{ Resolution (Rs) } \\
\hline & YMC & 16.47 & 0.85 & 4.32 & 0.45 & 2.89 & 0.03 \\
\hline & Shiseido & 16.24 & 1.16 & 3.23 & 0.79 & 2.17 & 0.03 \\
\hline & Sodex & 7.66 & 0.35 & 3.35 & 0.43 & 2.89 & 0.03 \\
\hline \multirow[t]{20}{*}{ Temperatu } & \multicolumn{7}{|c|}{ Theoretical plate (N) } \\
\hline & 25 & 1122 & 129 & 4146 & 143 & 7214 & 210 \\
\hline & 30 & 1183 & 109 & 4226 & 138 & 9217 & 597 \\
\hline & 35 & 1015 & 42 & 4183 & 129 & 4450 & 239 \\
\hline & 40 & 1003 & 49 & 3844 & 193 & 4540 & 64 \\
\hline & \multicolumn{7}{|c|}{ Capacity factor (k') } \\
\hline & 25 & 3.75 & 0.04 & 9.20 & 0.12 & 10.80 & 0.14 \\
\hline & 30 & 3.61 & 0.04 & 8.88 & 0.10 & 10.39 & 0.12 \\
\hline & 35 & 3.51 & 0.01 & 8.56 & 0.02 & 9.99 & 0.02 \\
\hline & 40 & 3.40 & 0.07 & 8.27 & 0.18 & 9.60 & 0.22 \\
\hline & \multicolumn{7}{|c|}{ Separation factor $(\alpha)$} \\
\hline & 25 & 0.49 & 0.00 & 1.21 & 0.01 & 1.17 & 0.00 \\
\hline & 30 & 0.48 & 0.01 & 1.17 & 0.02 & 1.16 & 0.00 \\
\hline & 35 & 0.46 & 0.00 & 1.13 & 0.00 & 1.17 & 0.00 \\
\hline & 40 & 0.45 & 0.01 & 1.09 & 0.02 & 1.16 & 0.00 \\
\hline & \multicolumn{7}{|c|}{ Resolution (Rs) } \\
\hline & 25 & 16.52 & 0.95 & 4.32 & 0.44 & 2.90 & 0.03 \\
\hline & 30 & 15.81 & 0.86 & 5.26 & 0.20 & 3.14 & 0.03 \\
\hline & 35 & 16.43 & 0.27 & 3.35 & 0.09 & 2.53 & 0.03 \\
\hline & 40 & 15.28 & 0.72 & 2.32 & 0.52 & 2.42 & 0.04 \\
\hline \multirow[t]{16}{*}{ Flow rate } & \multicolumn{7}{|c|}{ Theoretical plate $(\mathbf{N})$} \\
\hline & 0.9 & 1125 & 119 & 4166 & 143 & 7315 & 410 \\
\hline & 1.0 & 1120 & 125 & 4124 & 145 & 7244 & 410 \\
\hline & 1.1 & 1116 & 105 & 4093 & 139 & 7187 & 439 \\
\hline & \multicolumn{7}{|c|}{ Capacity factor $\left(\mathbf{k}^{\prime}\right)$} \\
\hline & 0.9 & 3.72 & 0.04 & 9.11 & 0.12 & 10.80 & 0.15 \\
\hline & 1.0 & 3.59 & 0.05 & 8.90 & 0.13 & 10.59 & 0.14 \\
\hline & 1.1 & 3.50 & 0.03 & 8.76 & 0.12 & 10.39 & 0.12 \\
\hline & \multicolumn{7}{|c|}{ Separation factor $(\alpha)$} \\
\hline & 0.9 & 0.49 & 0.03 & 1.21 & 0.01 & 1.25 & 0.02 \\
\hline & 1.0 & 0.47 & 0.02 & 1.16 & 0.02 & 1.19 & 0.01 \\
\hline & 1.1 & 0.45 & 0.02 & 1.13 & 0.02 & 1.16 & 0.01 \\
\hline & Resolutio & on (Rs) & & & & & \\
\hline & 0.9 & 16.88 & 0.72 & 4.42 & 0.52 & 3.11 & 0.04 \\
\hline & 1.0 & 16.43 & 0.75 & 4.22 & 0.40 & 2.99 & 0.03 \\
\hline & 1.1 & 15.91 & 0.86 & 4.16 & 0.20 & 2.74 & 0.03 \\
\hline
\end{tabular}

were maintained so there would not be any interference with the other peaks for the Cimicifugae Rhizoma. The experimental conditions, such as the column temperature, column species and mobile phases, were purposely altered. The theoretical plate $(\mathrm{N})$, capacity factor $\left(\mathrm{k}^{\prime}\right)$, separation factor $(\alpha)$ and resolution (Rs) were evaluated. To evaluate the suitability three different columns, YMC, Shiseido and Shodex, were compared with regard to four analytical factors $\left(\mathrm{N}, \mathrm{k}^{\prime}, \alpha\right.$ and $\left.\mathrm{Rs}\right)$ on the column temperature of $30^{\circ} \mathrm{C}$. The result showed that four analytical factors did not differ greatly, depending on the column species (YMC, Shiseido and Shodex). Four different column temperatures, 25, 30, 35 and $40{ }^{\circ} \mathrm{C}$, were compared with regard to four analytical factors using YMC column. The result showed that four analytical factors did not differ greatly, depending on the column temperature $\left(25,30,35\right.$ and $\left.40^{\circ} \mathrm{C}\right)$. Three different flow rates, $0.9,1.0$ and $1.1 \mathrm{~mL} / \mathrm{min}$, were compared with regard to four analytical factors using YMC column on $25^{\circ} \mathrm{C}$. The result showed that four analytical factors did not differ greatly, depending on the flow rates $(0.9,1.0$ and 1.1$)$. We optimized by changing the chromatographic parameters, but the four analytical factors did not differ greatly, therefore this experiment condition were sufficiently robust.

The sample stability test was determined with a standard mixture solution at $0,0.5,1,2,5,10,15$ and 30 days. During this period, the solution was stored at no light conditions, and at room temperature and $4{ }^{\circ} \mathrm{C}$, respectively. The resulting data indicated that all marker analytes remained stable more than 98\% during the experimental period.

Sample Analysis. The developed HPLC/UV method was then applied to the simultaneous determination of the three compounds, caffeic acid (1), ferulic acid (2) and isoferulic acid (3) in the Cimicifugae Rhizoma. Twelve authentic Cimicifugae Rhizoma samples corresponding to five different Cimicifugae Rhizoma species and seventeen commercially available Cimicifugae Rhizoma samples were obtained from Korea and China. The developed analytical method was subsequently applied to the simultaneous determination of the three components in Cimicifugae Rhizoma extract. The quantity of each compound present in samples was determined and the results are summarized in Table 5. Each sample was analyzed in triplicate to ensure the reproducibility of the quantitative result. The results indicated that, the contents $(\mathrm{mg} / \mathrm{g})$ of caffeic acid showed $1.5 \sim 3.5$ $(\mathrm{mg} / \mathrm{g})$ in C. foetida and C. simplex and $0.1 \sim 1.0(\mathrm{mg} / \mathrm{g})$ in $C$. heracleifolia, C. dahurica, C. foetida (mutation) and C. japonica. In the commercial samples, the assays of caffeic acid showed $0.1 \sim 0.4(\mathrm{mg} / \mathrm{g})$ except c25 $(0.9 \mathrm{mg} / \mathrm{g})$, corresponding to lower quantities than $C$. foetida and $C$. simplex. The contents of isoferulic acid showed $0.001 \sim 0.3(\mathrm{mg} / \mathrm{g})$ in $C$. foetida, $C$. simplex and C. japonica, and $1.6 \sim 4.6(\mathrm{mg} / \mathrm{g})$ in C. heracleifolia, C. dahurica and $C$. foetida (mutation). In the commercial samples, the contents of isoferulic acid showed $1.6 \sim 4.3(\mathrm{mg} / \mathrm{g})$, nearly the same as $C$. heracleifolia, $C$. dahurica and $C$. foetida (mutation). Additionally, the contents of ferulic acid showed $0.2 \sim 0.7$ $(\mathrm{mg} / \mathrm{g})$ in all of Cimicifugae Rhizoma species and commercial samples except c12 $(0.04 \mathrm{mg} / \mathrm{g})$ and c25 $(0.08 \mathrm{mg} / \mathrm{g})$. Consequently, $C$. foetida and $C$. simplex species samples were higher than C. heracleifolia, C. dahurica, C. foetida (mutation) and $C$. japonica species samples in the caffeic acid assays, whereas 
Table 5. Contents (mg/g) of phenolic components in Cimicifugae Rhizoma $(\mathrm{n}=3)$

\begin{tabular}{|c|c|c|c|c|c|c|c|c|c|}
\hline \multirow{3}{*}{ Sample } & \multicolumn{9}{|c|}{ Contents (mg/g) } \\
\hline & \multicolumn{3}{|c|}{ Caffeic aicd } & \multicolumn{3}{|c|}{ Ferulic aicd } & \multicolumn{3}{|c|}{ Isoferulic aicd } \\
\hline & Mean & SD & RSD & mean & SD & RSD & mean & SD & RSD \\
\hline $\mathrm{c} 1$ & 0.118 & 0.002 & 1.99 & 0.516 & 0.013 & 2.61 & 1.575 & 0.023 & 1.48 \\
\hline c2 & 0.149 & 0.001 & 0.13 & 0.658 & 0.002 & 0.28 & 2.237 & 0.007 & 0.30 \\
\hline c3 & 0.611 & 0.004 & 0.64 & 0.233 & 0.001 & 0.10 & 5.243 & 0.003 & 0.63 \\
\hline $\mathrm{c} 4$ & 1.018 & 0.015 & 1.50 & 0.373 & 0.004 & 1.08 & 2.520 & 0.039 & 1.56 \\
\hline $\mathrm{c} 5$ & 0.333 & 0.003 & 0.94 & 0.490 & 0.002 & 0.49 & 4.550 & 0.044 & 0.96 \\
\hline c6 & 0.592 & 0.007 & 1.13 & 0.305 & 0.001 & 1.13 & 3.457 & 0.006 & 0.18 \\
\hline c7 & 1.537 & 0.015 & 0.97 & 0.376 & 0.006 & 0.15 & 0.210 & 0.003 & 1.53 \\
\hline $\mathrm{c} 8$ & 3.454 & 0.028 & 0.80 & 0.320 & 0.002 & 0.56 & 0.001 & 0.002 & 1.95 \\
\hline c9 & 0.292 & 0.003 & 0.99 & 0.324 & 0.002 & 0.75 & 2.147 & 0.022 & 1.04 \\
\hline $\mathrm{c} 10$ & 2.054 & 0.001 & 0.06 & 0.329 & 0.005 & 1.57 & 0.063 & 0.002 & 3.17 \\
\hline c11 & 1.928 & 0.019 & 1.01 & 0.252 & 0.006 & 1.12 & 0.019 & 0.007 & 3.56 \\
\hline $\mathrm{c} 12$ & 0.555 & 0.014 & 2.45 & 0.039 & 0.001 & 2.74 & 0.298 & 0.008 & 2.66 \\
\hline $\mathrm{c} 13$ & 0.258 & 0.004 & 1.44 & 0.406 & 0.001 & 0.18 & 2.246 & 0.017 & 0.75 \\
\hline c14 & 0.389 & 0.002 & 0.48 & 0.453 & 0.001 & 0.07 & 2.205 & 0.001 & 0.07 \\
\hline c15 & 0.148 & 0.001 & 0.96 & 0.270 & 0.002 & 0.86 & 1.885 & 0.031 & 1.66 \\
\hline c16 & 0.222 & 0.004 & 1.79 & 0.344 & 0.010 & 1.01 & 2.263 & 0.022 & 0.96 \\
\hline c17 & 0.178 & 0.001 & 0.48 & 0.350 & 0.004 & 1.05 & 2.126 & 0.022 & 1.01 \\
\hline c18 & 0.151 & 0.001 & 0.99 & 0.220 & 0.001 & 0.34 & 1.566 & 0.007 & 0.48 \\
\hline c19 & 0.247 & 0.002 & 0.66 & 0.320 & 0.001 & 0.16 & 2.067 & 0.017 & 0.83 \\
\hline $\mathrm{c} 20$ & 0.189 & 0.002 & 0.99 & 0.291 & 0.002 & 0.64 & 2.329 & 0.017 & 0.72 \\
\hline c21 & 0.147 & 0.002 & 1.27 & 0.278 & 0.002 & 0.67 & 1.694 & 0.016 & 0.99 \\
\hline c22 & 0.215 & 0.001 & 0.91 & 0.325 & 0.002 & 0.57 & 1.859 & 0.020 & 1.06 \\
\hline $\mathrm{c} 23$ & 0.183 & 0.002 & 1.02 & 0.403 & 0.003 & 0.46 & 2.230 & 0.018 & 0.80 \\
\hline c24 & 0.225 & 0.002 & 0.89 & 0.393 & 0.002 & 0.48 & 2.309 & 0.021 & 0.91 \\
\hline $\mathrm{c} 25$ & 0.926 & 0.031 & 3.37 & 0.076 & 0.022 & 2.87 & 4.340 & 0.127 & 2.93 \\
\hline c26 & 0.346 & 0.008 & 2.24 & 0.573 & 0.012 & 2.01 & 3.132 & 0.069 & 2.20 \\
\hline c27 & 0.299 & 0.005 & 1.62 & 0.521 & 0.007 & 1.39 & 2.515 & 0.041 & 1.65 \\
\hline $\mathrm{c} 28$ & 0.378 & 0.013 & 3.50 & 0.429 & 0.012 & 2.76 & 2.483 & 0.101 & 4.09 \\
\hline c29 & 0.404 & 0.04 & 0.90 & 0.459 & 0.006 & 1.30 & 2.733 & 0.021 & 0.78 \\
\hline
\end{tabular}

C. heracleifolia, C. dahurica, and C. foetida (mutation) species samples were higher than $C$. foetida, $C$. simplex and $C$. japonica ones in the isoferulic acid assays. In the ferulic acid assay, we couldn't find any differences among species. In the quantitative analysis of Cimicifugae Rhizoma we indicated that, the Cimicifugae Rhizoma samples clustered two groups same as mentioned below. It was considered that $C$. japonica was clustered together with $C$. foetida and $C$. simplex due to a low quantity $(0.3 \mathrm{mg} / \mathrm{g})$ of isoferulic acid.

Pattem Recognition Analysis. To evaluate the phytochemical equivalency between the twenty nine samples corresponding to twelve authentic and seventeen commercial ones, pattern recognition analysis was conducted. In this study we used three marker compound peaks (caffeic acid (1), ferulic acid (2) and isoferulic acid (3)) for pattern recognition analysis. For pattern recognition analysis, three common peaks were selected based on the relative retention time for 4-hydroxycinnamic acid (I.S.) peak and used for a dataset. From the pattern analysis of Pam analysis (Fig. 3 and Fig. 4) and Hclust analysis (Fig. 5 and
Fig. 6) we indicated that, all of the samples were clustered to two groups (A, B). In authentic specimen analysis, we could build two clusters (A and B), and all of the species were successfully clustered two groups (A and B). In Pam analysis (Fig. 3) of twelve authentic specimen samples, we also found two clustering groups, exhibiting the same result as Pam analysis (Fig. 4). $\mathrm{C} 1 \sim \mathrm{c} 4$ (C. heracleifolia) and $\mathrm{c} 5$ and $\mathrm{c} 6$ (C. dahurica) samples belong to the group A, and $\mathrm{c} 7$ and $\mathrm{c} 8$ (C. foetida), c10 and $\mathrm{c} 11$ (C. simplex) and c12 (C. japonica) belong to the group B. A mutation sample of $C$. foetida, $\mathrm{c} 9$, was clustered with group A because of the contents of caffeic acid $(0.29 \mathrm{mg} / \mathrm{g})$ and isoferulic acid $(2.15 \mathrm{mg} / \mathrm{g})$. The contents of caffeic acid and isoferulic acid in C. foetida corresponding to Group B were $1.5 \sim 3.5 \mathrm{mg} / \mathrm{g}$ and $0.001 \sim 0.3 \mathrm{mg} / \mathrm{g}$, respectively. There are low caffeic acid and high isoferulic acid contents in Group A, whereas high caffeic acid and low isoferulic acid contents in Group B. Thus, the contents of caffeic acid and isoferulic acid in sample were important factors to build two clusters, A and B. In Pam analysis (Fig. 4) the commercial samples (c13 c29) were clustered 


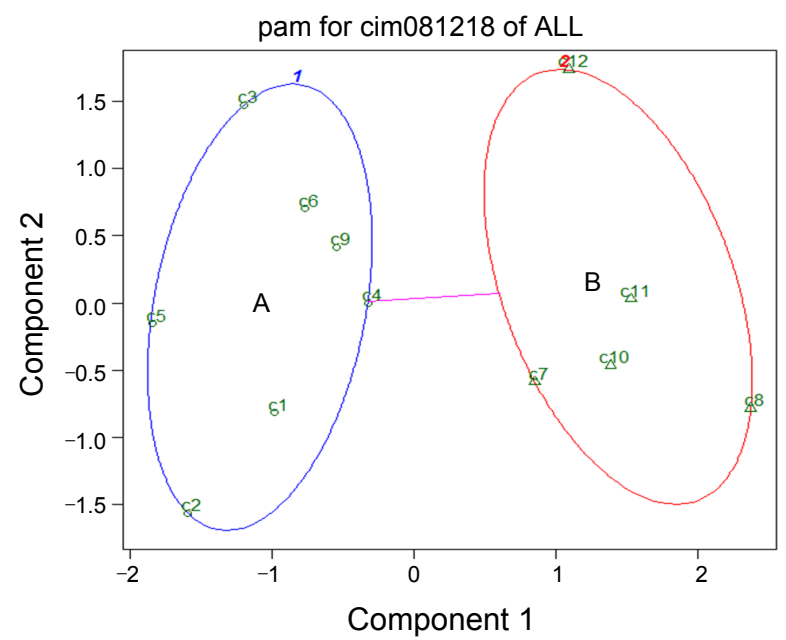

These two components explain $86.92 \%$ of the point variability.

Figure 3. Pam of 12 authentic specimens of Cimicifugae Rhizoma. A: (C. heracleifolia and C. dahurica), B: (C. foetida, C. simplex and C. japonica)

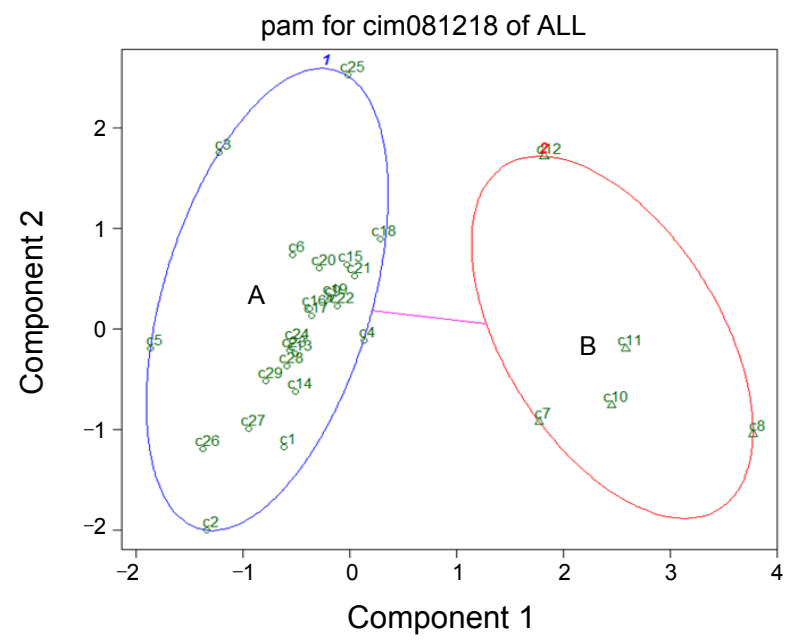

These two components explain $83.43 \%$ of the point variability.

Figure 4. Pam of 29 specimens of Cimicifugae Rhizoma including 17 commercial samples. A: (C. heracleifolia and $C$. dahurica), B: (C. foetida, C. simplex and C. japonica)

together with the group A. The commercial samples were purchased at different market and have different producer, but c13 c29 were clustered with group A. Therefore, all of the commercial samples were $C$. heracleifolia, or $C$. dahurica. Therefore, this result demonstrated that pattern recognition analysis can provide more comprehensive information for the chemical equivalency which can be omitted in the general simultaneous quantitative analysis. Thus, the pattern analysis result will be used to check the quality control of Cimicifugae Rhizoma.

\section{Conclusions}

A rapid and optimized chromatographic method with UV detection was designed for the quality control of Cimicifugae Rhizoma, well-known Korean traditional medicine. Validation

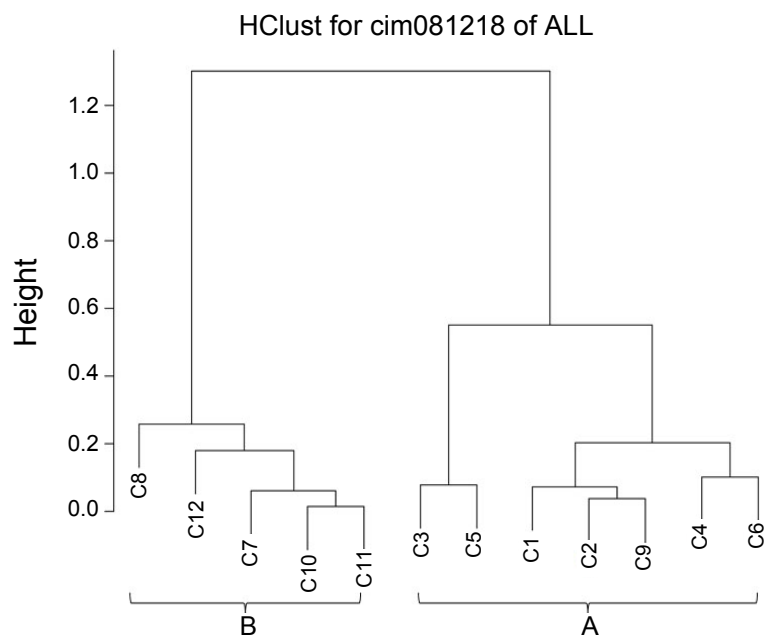

Figure 5. Hclust of 12 authentic specimens of Cimicifugae Rhizoma. A: (C. heracleifolia and C. dahurica), B: (C. foetida, C. simplex and C. japonica)

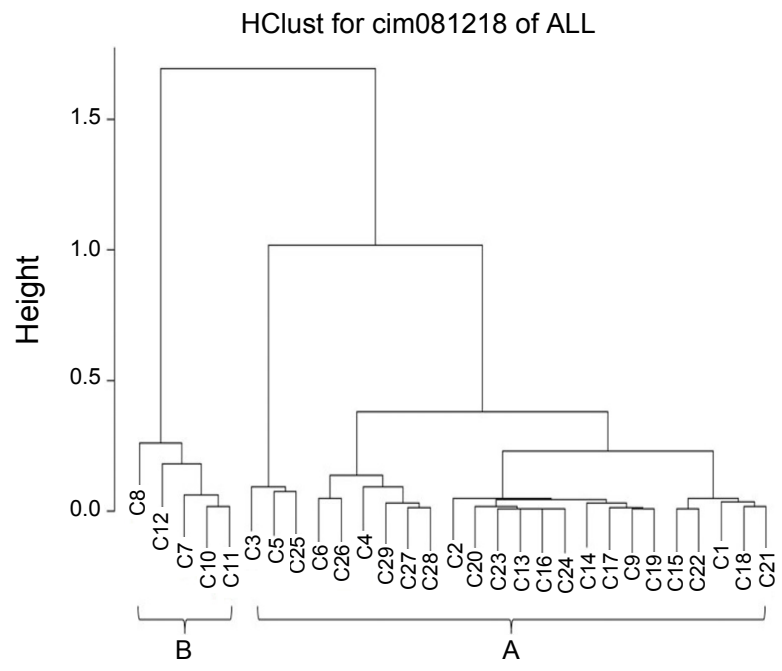

Figure 6. Hclust of specimens of Cimicifugae Rhizoma including 17 commercial samples. A: (C. heracleifolia and $C$. dahurica), B: (C. foetida, C. simplex and C. japonica)

data indicates that the developed analytical methods are suitable to measure the concentration of three compounds to apply to pattern recognition analysis of Cimicifugae Rhizoma. The developed HPLC/UV method for quantitative analysis of major bioactive compounds, along with a pattern-recognition method, can provide the promising prospect to comprehensive quality control of Cimicifugae Rhizoma and its related herbal medicine.

C. foetida and C. simplex species samples were higher than C. heracleifolia, C. dahurica, C. foetida (mutation) and C. japonica species samples in the caffeic acid assays, whereas $C$. heracleifolia, $C$. dahurica, and $C$. foetida (mutation) species samples were higher than $C$. foetida, $C$. simplex and $C$. japonica ones in the isoferulic acid assays. In the ferulic acid assay, we couldn't find any differences among species. Our results confirm that caffeic acid can serve as the species-specific marker 
compounds to distinguish authentic C. foetida and C. simplex, and isoferulic aicd to distinguish authentic $C$. heracleifolia and C. dahurica species in a pattern-recognition analysis. Therefore it is considered that caffeic acid, ferulic acid and isoferulic acid are adequate as marker compounds of quality control to distinguish the different species of Cimicifugae Rhizoma. In the pattern recognition analysis we indicated that, all of the samples were clustered to two groups A (C. heracleifolia and C. dahurica $)$ and $\mathrm{B}$ (C. foetida, C. simplex and C. japonica), and the commercial samples (c13 c29) purchased from the markets were clustered together with group A. Therefore, the commercial samples were C. heracleifoia or C. dahurica.

Acknowledgments. This work was supported by a grant from the Korea Food and Drug Administration for Studies on Standardization of Herbal Medicine (2007).

\section{References}

1. Islam, M. N.; Lee, S. K.; Jeong, S. Y.; Kim, D. H.; Jin, C.; Yoo, H. H. Bull. Korean Chem. Soc. 2009, 30, 137.

2. Ajisawa, Y.; Kitazawa, M.; Uchida, M.; Kobayashi, M. Yakugaku
Zasshi 1996, 116, 50 .

3. Noguchi, M.; Nagai, M.; Koeda, M.; Nakayama, S.; Sakurai, N.; Takahira M.; Kusano, G. Biol. Pharm. Bull. 1998, 21, 1163.

4. Kruse, S. O.; Lohning, A.; Paull, G. F.; Winterhoff, H.; Nahrstedt, A. Planta Med. 1999, 65, 763.

5. Sakai, S.; Kawamata, H.; Kogure, T.; Mantani, N.; Terasawa, K.; Umatake, M.; Ochiai, H. Mediators Inflamm. 1999, 8, 173.

6. Watanabe, K.; Mimaki, Y.; Sakagami, H.; Sashida, Y. Chem. Pharm. Bull. 2002, 50, 121.

7. Lin, S. J.; Chen, C. S.; Lin, S. S.; Chou, M. Y.; Shih, H. C.; Lee, I. P.; Kao, C. T.; Ho, C. C.; Chen, F. L.; Ho, Y. C.; Hsieh, K. H.; Huang, C. R.; Yang, C. C. Food Chem. Toxicol. 2006, 44, 2078.

8. Qiu, M.; Kim, J. H.; Lee, H. K.; Min, B. S. Phytother. Res. 2006, 20 , 945.

9. Jiang, B.; Yang, H.; Nuntanakorn, P.; Balick M. J.; Kronenberg, F.; Kennelly, E. J. J. Ethnopharmacol. 2005, 96, 521.

10. Jiang, B.; Kronenberg, F.; Balick, M. J.; Kennelly, E. J. Phytomedicine 2006, 13, 477.

11. He, K.; Pauli, G. F.; Zheng, B.; Wang, H.; Bai, N.; Peng, T.; Roller, M.; Zheng, Q. Y. J. Chromatogr. A 2006, 1112, 241.

12. Lin, L. C.; Kuo, Y. C.; Chou C. J. J. Nat. Prod. 1999, 62, 405.

13. Wandji, J.; Nkengfack, A. E.; Fomum, Z. T.; Ubillas, R.; Killday, K. B.; Tempesta, M. S. J. Nat. Prod. 1990, 53, 1425.

14. Usia, T.; Banskota, A. H.; Tezuka, Y.; Midorikawa, K.; Matsushige, K.; Kadota, S. J. Nat. Prod. 2002, 65, 673. 